\title{
Analysis on the Settlement of Adjacent Buildings Caused by the Underpassing Construction of the Biased Tunnel
}

\author{
Xu Zhang ${ }^{1,}{ }^{*}$,Dong Wang ${ }^{1}$, Tong $\mathrm{Wu}^{2}$, Zhenyue $\mathrm{Shi}^{1}$,Shaobo $\mathrm{Li}^{1}$, Yuanyuan $\operatorname{Tian}^{1}$ and Hongyue You ${ }^{1}$ \\ ${ }^{1}$ Shandong University of Science and Technology, 579 Qianwangang Road, Huangdao District, Qingdao City, Shandong, China \\ ${ }^{2}$ Jinan First Construction Group Co., Ltd. 295 North Industrial Road, Licheng District, Jinan City, Shandong, China
}

\begin{abstract}
Through the simulation analysis of the settlement and deformation law of the surface buildings caused by the construction of the biased tunnel, the following points are obtained: (1) The Peak formula is revised, and the influence range of the biased tunnel is predicted based on the formula. (2) It is concluded that when the tunnel is biased, the position of maximum deformation caused by ground settlement is generally in a parallel area 0.5 times the buried depth from the center line of the tunnel. (3) Through the double-layer verification of simulation analysis and monitoring measurement data, prior to the construction of buildings with similar weak foundations, their foundations should be reinforced in advance. (4) In the process of this simulation, the complicated influence of water pressure on tunnel excavation was not considered, which can be further optimized in the later stage.
\end{abstract}

\section{Introduction}

The tunnel excavation process will have many adverse effects on the surrounding environment. Especially in the excavation process of urban undercut tunnels, the underpass construction of the tunnel will cause deformation of the overlying soil layer above it, which will reflect the ground settlement on the ground surface. The foundation of the building above will also be affected. In severe cases, it will cause the foundation to crack, and the building will sink or even settle unevenly, endangering the use of the building.

When studying the impact of tunnel excavation on the surrounding environment, most researchers use numerical simulation methods for research and analysis. Numerical simulation has been widely used in the industry due to its accuracy, high reducibility, and low cost. Fruitful results have been achieved in China. At this stage, with regard to the settlement caused by the tunnel underpass construction, Zhou Zhi and others ${ }^{[1]}$ have studied the deformation law of the upper frame structure building caused by the double-line undercut tunnel during the underpass construction through simulation analysis; Liu Houxiang et $\mathrm{al}^{[2]}$ studied the settlement laws of buildings caused by tunnels under different locations through the numerical analysis of dense small brick-concrete buildings; Gong Zhiqun and others ${ }^{[3]}$ used engineering examples to analyze the superimposed impact of tunnel group construction on nearby buildings; Qi Taiyue ${ }^{[4]}$ used FLAC3D to establish a three-dimensional numerical model and studied the characteristics of ground settlement caused by subway construction; Tian Siming et a ${ }^{[5]}$ conducted research and analysis on the interaction between railway tunnel construction and adjacent buildings, and evaluated the effects of various engineering measures adopted in construction; Liu Huilin, Shen Xingzhu and others ${ }^{[6-7]}$ conducted simulation analysis on the settlement of the above buildings caused by the mining method and shield method tunnel construction, and verified their respective settlement laws caused by different construction methods; Zhu Caihui et al ${ }^{[8]}$ analyzed the ground settlement caused by tunnel construction based on the Peck formula, which further laid a theoretical foundation for studying the settlement laws of surface buildings.

Existing research has carried out detailed analysis on the settlement reasons, settlement mechanism, and settlement effects of adjacent buildings caused by tunnel construction, and has obtained relatively mature theoretical results. At the same time, in the research field of biased tunnels, the law and principle of surface settlement caused by the underpass construction of the bias tunnel has also entered a relatively mature stage. However, there is still a lack of research on the impact of the underpass construction of a biased tunnel on adjacent buildings.

Here, we are going to adopt the method of combining theory and practice, combined with engineering examples, and use FLAC3D to establish a numerical model to carry out the research on the impact of the underpass construction of the biased tunnel on the adjacent buildings. This research can enrich the existing tunnel excavation. The research content also provides reference for similar projects.

*Corresponding author: 201982040021@sdust.edu.cn 


\section{Project Overview}

The interval between No. 1 inclined shaft and No. 22 well in the fourth work area, GFHD-2 Standard, GuangzhouFoshan East Ring Road, is constructed by mining method, Starting and ending mileage: DSK33+860 DSK35+230, Among them, the tunnel uplink DSK34+090 DSK34+436 passes through Guangzhou Youhao Senior Apartment. The DSK34+370 DSK34+435 section of the tunnel upward line runs along the foot of the mountain slope, The side above the tunnel is a mountain with a slope ratio of 1:0.75; on the other side are two buildings, which are 9 and 7 floors in the order of distance from the tunnel. The house was built in the late 1990s and the foundation was expanded. The foundation was buried at a depth of about $2 \mathrm{~m}$, and the foundation was weak. The detailed location distribution and monitoring point layout are shown in Figure 1.

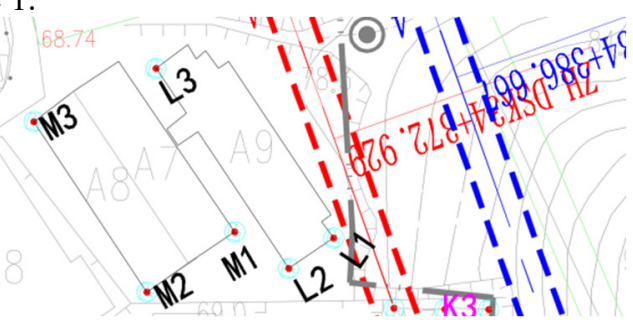

Figure 1. Layout of monitoring points in elderly apartments.

\section{Establishment of numerical simulation model}

\subsection{Tunnel parameters}

Take the buried depth of the tunnel as $40 \mathrm{~m}$, and take $60 \mathrm{~m}$ from the ground downward in the vertical direction. The midline side of the tunnel along the strike direction is a hillside with a slope ratio of 1:0.75. The maximum height of the hillside is $20 \mathrm{~m}$, and the distance in the horizontal direction of the tunnel section is $150 \mathrm{~m}$. Along the center line of the tunnel, the left and right sides are $75 \mathrm{~m}$ on each side. The two rows of parallel buildings form an angle of $13^{\circ}$ with the direction of the tunnel. The clear height of the tunnel is $9.38 \mathrm{~m}$, and the width of the road baseline is $7.06 \mathrm{~m}$.

When constructing the finite element grid model, the tunnel is centered and the grid is constructed radially to facilitate more intuitive observation of the stress and strain caused by the tunnel excavation construction on the surface buildings. The boundary conditions are set as follows: add horizontal and vertical directions at the bottom Constraints, horizontal constraints are added to the four sides, no vertical constraints are added, and the ground surface is a free surface where settlement can occur.

\subsection{Building parameters}

The building is two parallel civil buildings with a frame structure. The foundation is an extended foundation, with a width of about $1.5 \mathrm{~m}$ and a foundation buried depth of $2 \mathrm{~m}$. The building closer to the tunnel has 9 floors, length $\times$ width $\times$ height: $45 \mathrm{~m} \times 10 \mathrm{~m} \times 30 \mathrm{~m}$, the other house has 7 floors, length $\times$ width $\times$ height: $38 \mathrm{~m} \times 19.5 \mathrm{~m} \times 24.5 \mathrm{~m}$, the distance between the two houses is $4.5 \mathrm{~m}$, the physical and mechanical parameters of the building materials are shown in Table 1. The image location diagram is shown in Figure 2.

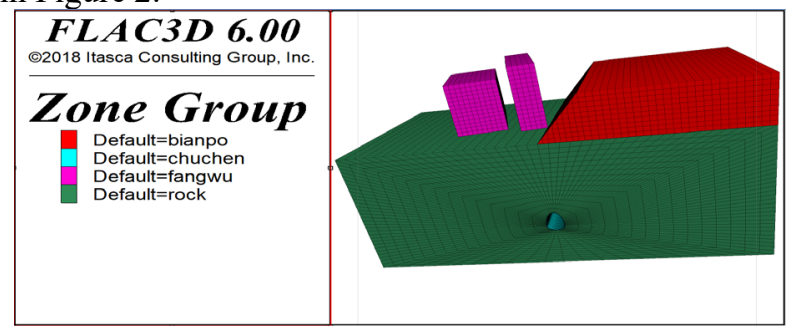

Figure 2. Tunnel-building numerical analysis model network diagram.

\subsection{Soil parameters}

According to the geological exploration of this geological section, the soil layer of this section is mainly mixed fill, silty clay, and weathered granite. Based on the geological exploration, the advanced drilling exploration in the tunnel reacts with the actual excavation conditions. The surface buildings are located in the foothills of the old time, and the underground is mostly loose sediments, and the depth of sediment accumulation exceeds the buried depth of the tunnel. Therefore, in the calculation, the soil layer can be simplified as a homogeneous soil layer for calculation. Because the groundwater system is relatively complex, the influence of the groundwater level on the soil layer is not considered for the time being. The constitutive relationship of the soil layer adopts the Moore-Coulomb model. The physical parameters of the soil layer are calculated according to the grade $\mathrm{V}$ surrounding rock, and the detailed parameters are shown in Table 1.

Table1. Material physical and mechanical parameters.

\begin{tabular}{llllllll}
\hline \multirow{2}{*}{ Parameter } & $\begin{array}{l}\text { Elastic Modulus } \\
\mathrm{E}(\mathrm{MPa})\end{array}$ & $\begin{array}{l}\text { Poisson's } \\
\mu\end{array}$ & $\begin{aligned} \text { ratio } \\
\end{aligned}$ & $\begin{array}{l}\text { Severe } \\
(\mathrm{kN} / \mathrm{m} 3)\end{array}$ & $\begin{array}{l}\text { Cohesion } \\
\mathrm{c}(\mathrm{kPa})\end{array}$ & $\begin{array}{l}\text { Internal } \\
\text { friction angle }\end{array}$ & $\begin{array}{l}\text { Dilatancy } \\
\text { angle } \psi\left({ }^{\circ}\right)\end{array}$ \\
\hline Soil layer & 100 & 0.35 & 20 & 30 & 22 & 8 \\
Initial lining & 25000 & 0.2 & 25 & $/$ & $/$ & $/$ \\
Wall & 200 & 0.1 & 20 & $/$ & $/$ & $/$ \\
Floor & 28000 & 0.2 & 25 & $/$ & $/$ & $/$ \\
Basis & 25000 & 0.2 & 25 & $/$ & $/$ & $/$ \\
\hline
\end{tabular}




\subsection{Numerical simulation program}

The main purpose of this simulation is to analyze the impact of the underpass construction of a biased tunnel on the settlement of adjacent buildings. The maximum settlement generally occurs after the excavation of the tunnel before the secondary lining is completed. Therefore, we ignore the impact of the tunnel body here. During the excavation process, and because the tunnel face of the right line of the tunnel has not yet entered the simulation range during the construction process where the left line of the tunnel passes underneath, the right line is ignored here and only the construction of the left line is considered.

\section{Simulation of settlement deformation analysis}

Assign the material physical and mechanical parameters provided in the previous section to the finite element model constructed by FLAC3D. After calculation, the following stress cloud diagram is obtained. It can be seen from Figure 3 that both buildings have a large range of vertical displacement. Figure 4 shows the simulated building. The displacement monitoring points of, their point numbers 1-6 correspond to the actual monitoring points L1-M3 respectively. From Figure 4, it can be seen that the average settlement of the buildings close to the center line of the tunnel is $38 \mathrm{~mm}$, and the average settlement of the buildings far away from the center line of the tunnel It can be seen from the curve trend that the settlement is still increasing, and the overall vertical displacement value exceeds the design control value $(30 \mathrm{~mm})$; At the same time, it can be seen from the figure that the mountains above the center line of the tunnel and to the right of the center line have produced greater deformation, and the deformation began to intrude into the tunnel body in a small vertical range; the surface area outside the building on the left side of the tunnel center line, The amount of settlement is small. Obviously, the impact on the surface settlement above the tunnel during the undercut tunnel construction is completely different from the impact on the building above the tunnel. In the past, the law of research on the surface settlement above the tunnel caused by the biased tunnel construction is in the building The area of settlement is not applicable; Although this model does not consider the groundwater level, the design of the excavation process is a one-time excavation, and the design of each parameter is relatively conservative, which will cause the building to produce a larger settlement deformation than the actual settlement value, but the design is actually used to guide the construction During the process, we still have to reserve a sufficient safety factor. Therefore, in the tunnel excavation process of this type of working condition, we should pre-reinforce the foundation of the building to ensure safe construction.

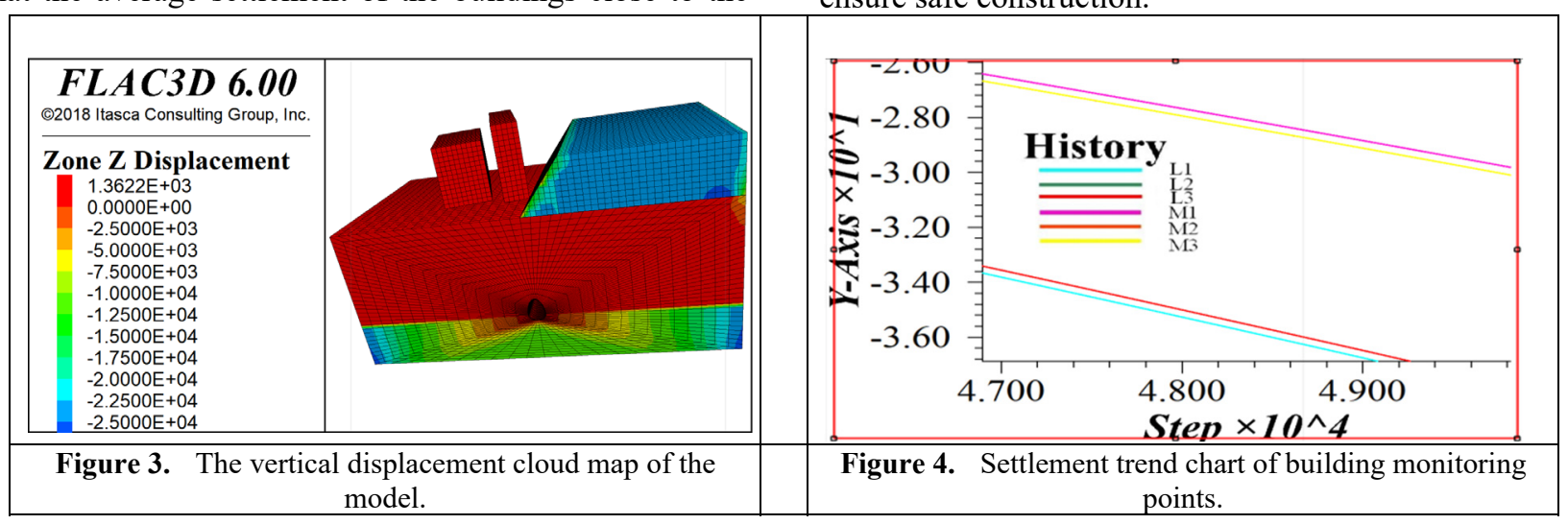

It can be seen from Figure 5 that the maximum stress concentration position of this model is the tunnel lining surface, and the stress on the tunnel lining surface is distributed in a beam-like discontinuous manner. It conducts force analysis; The direction of tunnel excavation in the figure is from the surface of the figure to the inner side of the figure. For the surface stress, it can be seen that although the surface stress is small, the tensile stress and the compressive stress cross in a strip shape, and the direction is perpendicular to the direction of the building, indicating that the building is in Under this kind of working condition, part of the surface shear stress will be affected, which is easy to cause shear deformation of the building and cause cracks in the main body of the building. At the site of this project, such cracks rarely appear in the building, which also confirms the good integrity of the building. 


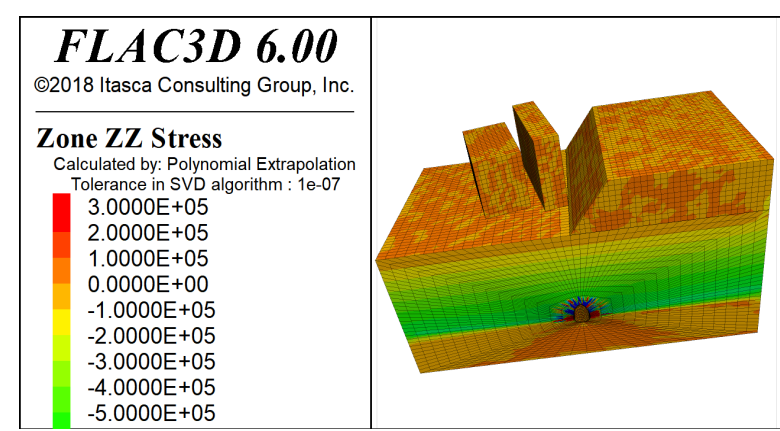

Figure 5. Vertical stress cloud diagram of the model.

Figure 6 shows the vertical stress cloud diagram of the model obtained by sparse grids on the basis of Figure 5 and lowering the convergence criteria. This stress cloud diagram has lost a greater guiding significance for the settlement of the actual building, but it can be seen from the image, The stress concentration area on the weak pressure side of the bias tunnel after finally converging is in the strip-shaped area 0.5 times the buried depth of the tunnel centerline. In this simulation condition, the area is just in the middle of the two buildings, and the stress direction is positive, indicating that the settlement value of the building in the strip area should be slightly larger than the settlement value on the other side, that is, two buildings The object is partially tilted toward the middle.

\section{Monitoring data verification}

By improving the Peak formula, assuming that the tunnel's influence range is 5 times the diameter of the tunnel, the settlement law at any point of the stratum affected by the biased tunnel can be calculated as:

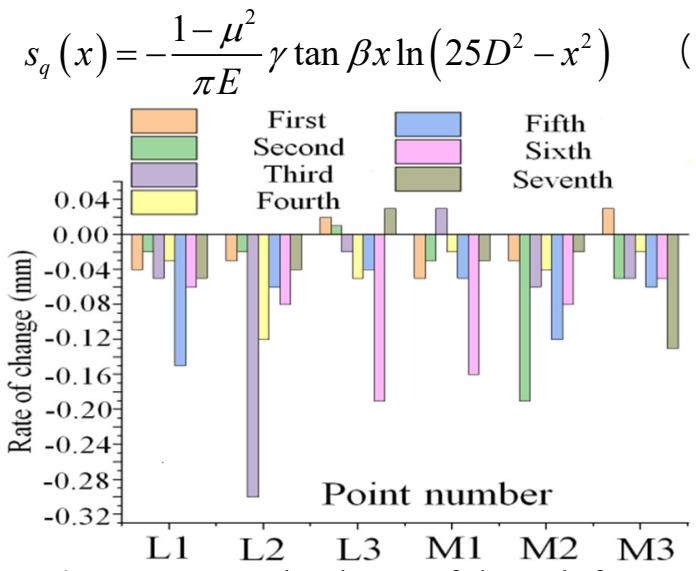

Figure 7. Measuring the rate of change before excavation.

After the tunnel face enters the house-affected area, the building begins to produce large settlements. When the tunnel is excavated to the closest position to the building at the center line, the tunnel face mileage is DSK34+357 at this time, the building's L1 point The maximum settlement rate is generated, and the settlement rate exceeds the warning value. Subsequently, as the tunnel is continuously excavated, the cumulative settlement value of the building continues to increase. When the tunnel face is flush with the rear of the building, it ushered in a larger

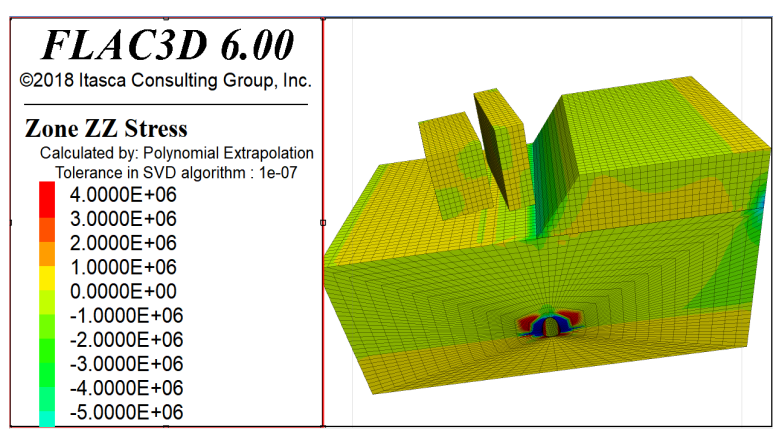

Figure 6. The vertical stress cloud diagram of the model after deepening calculation.

In the formula: $s_{q}(x)$ is the surface settlement under the bias load; $E$ is the compressive modulus of the formation; $u$ is Poisson's ratio; $\eta_{1}$ and $\eta_{2}$ are respectively the integration interval, which is the surface interval of the tunnel's influence range; $\beta$ is the bias angle; $\gamma$ is the formation severity.

In order to provide a guarantee for safe construction, before the tunnel face reaches the affected area of the surface building, the building settlement observation point is set up on the apartment building for the elderly, and the building settlement observation is started at the same time.

After the previous data collection, some house settlement data was obtained. The data showed that when the tunnel face did not enter the affected area, the settlement value of the two buildings was relatively small, and the foundation of the house was in a relatively stable state. , The rate of change of a single measurement does not exceed $0.3 \mathrm{~mm} /$ time, and the maximum accumulated settlement is $-0.67 \mathrm{~mm}$, see Figure 7 and Figure 8 for details.

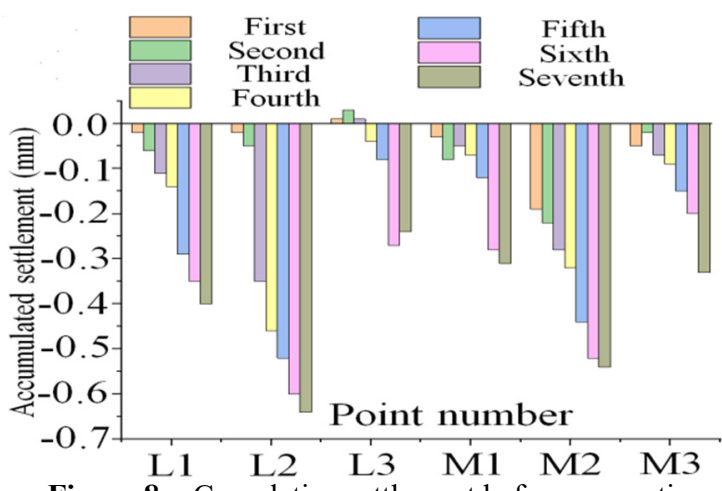

Figure 8. Cumulative settlement before excavation.

The rate of settlement changes, and eventually the building stabilizes. According to the analysis of the monitoring data and drawings, it can be concluded that the maximum amount of settlement occurred at the points L3 and M1 between the two buildings, and the maximum settlement value reached about $23 \mathrm{~mm}$. The cumulative settlement of the remaining points was basically symmetrical. As a result, It shows that the settlement trend of the two buildings is inclined between the two buildings, and uneven settlement occurs. This result corresponds to 
the above-mentioned numerical simulation result. The detailed data is shown in Figure 9 and Figure 10.

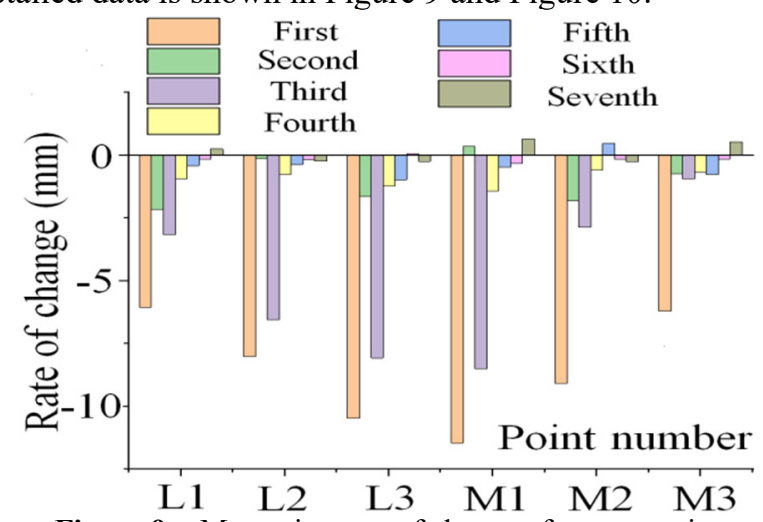

Figure 9. Measuring rate of change after excavation.

\section{Conclusion}

After analysis, the following conclusions can be drawn:

(1) Using the modified Peak formula to derive the influence range of the bias tunnel. Compared with the settlement change law of the actual building foundation, the influence range deduced by the formula has more accurate guiding significance.

(2) Above the biased tunnel, the maximum settlement deformation occurs in the area 0.5 times the depth of the tunnel center line. In the design of the tunnel, the calculation of such areas should be strengthened to ensure safe construction.

(3) Through numerical simulation and actual monitoring and measurement data analysis, for buildings with weak foundations, during the underpass construction of the tunnel, the settlement rate and cumulative settlement of the house exceeded the warning value in a small range, such buildings should be reinforced in advance during excavation and construction.

(4) In the numerical simulation of this paper, the water pressure was not considered, which caused a partial deviation between the simulated value and the monitored value. The model still needs to be improved in the later stage.

\section{References}

1. Zhou Zhi,Heng Chaoyang,Sun Xiyuan.Research on deformation laws of frame structure buildings induced by tunnel underpass construction[J].Chinese Journal of Geotechnical Engineering,2015,37(S1):110-114.

2. Liu Houxiang,Ren Zhiyong,Chen Siyu.Analysis of the deformation of adjacent buildings caused by tunnel underpassing at different positions[J].China Civil Engineering Journal,2014,47(08):128-137.

3. Gong Zhiqun, Tang Cong,Gong Yijun,Li Yang,Gao Dongbo.Study on the superimposed influence of foundation pit and tunnel group construction on adjacent buildings[J].Chinese Journal of

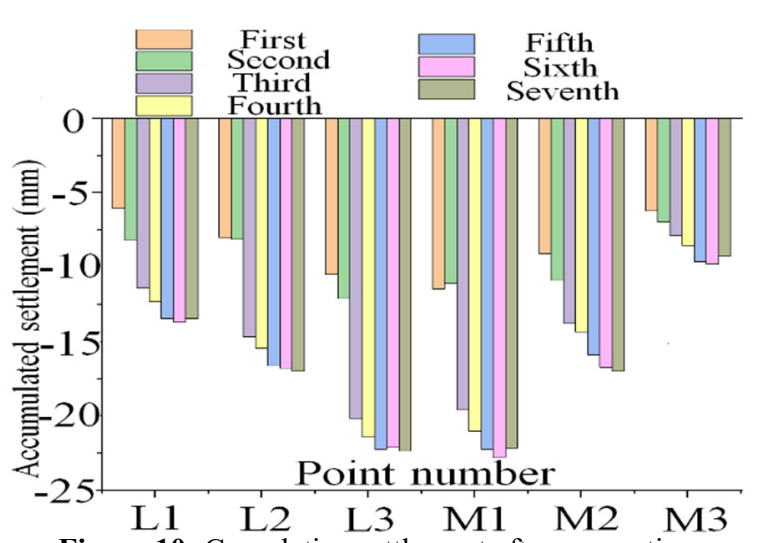

Figure 10. Cumulative settlement after excavation.

Underground Space

and

Engineering,2020,16(S2):752-761.

4. Qi Taiyue.Study on characteristics of ground and building settlement caused by subway construction[J].Chinese Journal of Geotechnical Engineering,2012,34(07):1283-1290.

5. Tian Siming,Huang Sheng, Chen Libao.Study on the Interaction between Intercity Railway Tunnel and Adjacent Buildings[J].Modern Tunnel Technology,2012,49(03):67-76.

6. Liu Huilin,Wang Dongxing.Study on the Change Law of Settlement of Undercut Metro Tunnels Passing Existing Buildings[J].Urban Rail Transit Research,2019,22(06):47-51.

7. Shen Xingzhu,Gao Feng,Wang Shaopeng, Yu Qingyang,Wang Shimin.Study on the influence of shield tunnel construction on shallow foundation buildings[J].Construction Technology,2017,46(13):132-137.

8. Zhu Caihui,Li Ning.Estimation method and law analysis of ground settlement induced by tunnel construction[J].Rock and Soil Mechanics,2016,37(S2):533-542. 